\section{IN THIS ISSUE:}

\section{What Are Characteristics of Significant Research?}

$\mathrm{W}$ hy does some research garner attention while other research goes almost entirely unnoticed or is quickly forgotten? What characteristics typify research that gets discussed, applied, scrutinized, denied, confirmed, and referenced in collegial conversations, popular publications, journal articles, and dissertations year after year? What are the characteristics of significant research?

Attempts to identify qualities that make research significant are not new. For example, in 1971 Davis presented a "Sociology of the Interesting" that he developed by analyzing research theories in the social sciences that caught the public's fancy and achieved wide circulation outside the limits of his discipline. Driven by the conviction that "what is needed is not more social theories, but more interesting social theories," Davis sought to identify features that these theories shared - features that made them not only interesting but also enduring.

The 27 researchers who participated in the Research Think Tank at the 1994 Association for Business Communication $(\mathrm{ABC})$ conference in San Diego undertook a similar task. As reported in a previous Doing Research column (March 1995), Think Tank participants represented the diversity of scholarly work in business and management communication and comprised well-known researchers among our Association membership, including research-award winners, past and present $\mathrm{ABC}$ officers, and journal editors, associate editors, and board members. This column provides a preliminary analysis of the list of characteristics of significant research they originated.

\section{How Was the "Characteristics of Significant Research" List Originated?}

The list of characteristics of significant research compiled by Think Tank participants resulted from a series of organized brainstorming activities which took the better part of a day to complete. Participants initially worked in small groups which had been determined prior to the event so that researchers with

Authors' Note: The authors thank Judy Lease for helping to compile the data and the ABC Research Think Tank participants who originated the "characteristics of significant research" list that is the basis for this column and whose names are listed in the March 1995 column.

\author{
Priscilla S. Rogers \\ University of Michigan, Ann Arbor
}

\section{John C. Sherblom}

University of Maine, Orono

diverse scholarly interests and publications would work together.

To begin, each group generated an exhaustive list of characteristics of significant research with each group member contributing in an "around-the-table" manner. If a group member could not think of a characteristic, he or she could pass a turn. Individual group members were to find inspiration for their individual contributions by recalling the features of sources that had been significant for their personal research endeavors, sources they had been asked to write on index cards just prior to the group activities but which they were not to reveal until the end of the event (see Rogers, "What Sources Have Inspired Our Research?" Business Communication Quarterly, March 1995).

Throughout the brainstorming process, characteristics were recorded on flipchart sheets. The goal of this initial activity was to generate as many individual characteristics of significant research as possible.

Next each group used a cut-and-tape process to separate and reassemble the individual characteristics into logical categories which they then labeled. While the groups enjoyed lunch, their categorized lists were exchanged. When the groups returned to work, they were to review and refine the lists they were given, lists which had been generated by other groups. This process involved rearranging characteristics, collapsing categories, and sometimes even giving away characteristics to another group. When a group felt comfortable with a list they taped it to the wall. By the end of this activity, 24 lists had been taped up around the room. Subsequently, the entire group of Think Tank participants reviewed together each of the 24 lists and merged them under 9 major categories.

\section{What Is the "Characteristics of Significant Research" List?}

The list of the characteristics of significant research resulting from the Think Tank consists of 293 individual entries, each of which appears under one of the following nine categories: outcomes, generative quality, methodology, expressing, attacks on status quo, authorial presence/ethos, syntheses, indexes of quality, and finding connections. Most of the entries or characteristics are sets of words or phrases that range from 3 
to 10 words in length as seen in the sample below taken from the Generative Quality category:

Changes theories of the world

Potential for lifetime of investigation

Encourages more discovery

Feels unfinished

Provocatively wrong

Convinces you that what it delivers is worth having

May include oxymorons or paradoxes (fruitful)

Speculative

Stimulate the imagination

Helps us re-see the familiar

Has an "ah ha!" element

Creates knowledge (new knowledge)

The longest entry, with 24 words, reads:

Frame within a frame - reflexive. Researchers researching subjects researching themselves (Example: Article

based on today's event. Example: Subject gives protocol, then comments on it.)

Only 54 or one-sixth of the entries are single words, including: "scholarly," "analytical," "theoretical," "contextualized," "historical," "multifaceted," "accessible," "unpretentious," "ambiguity," "relevant," "timeless," "insightful," "provocative," "pace-setter," "energetic," "cranky," "angry," "speculative," "smart," "fun," "weird," "poetic," and "Eureka!" Taken together, the categories and characteristics comprise a text of 1,059 individual words. (This text is available from the authors upon request).

\section{What Does the "Characteristics of Significant Research" List Tell Us?}

To examine the characteristics of significant research, we employed several types of content analysis: semantic network and cluster analyses using the CATPAC (Woelfel, 1990) and traditional descriptive and syntactical analyses (Krippendorff, 1980). Findings reported here focus on those words that are important by the frequency of their occurrence as identified using semantic network analysis. This focus is based on the assumption that words represent semantic concepts and, following an activation approach to cognition, the more frequently a word occurs in the text the more active the concept it represents and the more important that concept is considered to be to the overall construct or conceptualization under investigation. Subsets of the most frequently occurring words were examined using textual analysis and dimensional network analysis; cluster analysis allowed us to see word relationships. Comparing the results of these different analyses revealed various co-occurrences or themes within the text and facilitated some preliminary conclusions.

\section{Frequently Occurring Words and Their Contextual Interpretation}

Semantic network analysis or perceptual mapping, is a quantitative procedure that allows words and associations among them to emerge that can then be inter. preted qualitatively (Carley \& Kaufer, 1993; Rice \& Danowski, 1993). The first step of the semantic network analysis was a word-frequency count that identified those words deemed important by their frequency of occurrence in the text as a whole. The complete text of 1,059 words (tokens) consists of 705 uniquely different words (types). Of these uniquely different words, 516 occurred only once, 119 occurred twice, and 70 occurred three or more times.

Semantic network analysis helps focus attention on a small number of concepts identified as important by their frequency of occurrence, but exactly how far to "zoom out" or "zoom in" the focusing lens to include more or fewer concepts in the analysis is a researcher decision. For this analysis, we initially zoomed in on the 70 uniquely different words shown in Table 1, first looking at the context of those words occurring more than 5 times to gain some understanding of the nature of the text as a whole.

As Table 1 reveals, words occurring more than five times include: "knowledge" (13), "new" (12), "based" (11), "data" (9), "makes" (7), "questions" (6), "interdisciplinary" (6), and "ideas" (6). We further interpreted these words by examining their textual context.

One might expect a list of characteristics of this kind to consist almost entirely of descriptive adjectives or nouns; however, that is not the case. Actually, the most frequently occurring words are found in entries dominated by verbs, suggesting that significant research involves "activity."

More than any other word, "knowledge" appears 13 times and is distributed evenly in just over half or 5 of the 9 categories. Analysis of the entries in which "knowledge" appears suggest that significant research is both built on knowledge (of methodology, of other disciplines, and from experience) and is itself the activity of creating or transforming knowledge. In fact, more than half of the entries in which "knowledge" appears include a verb as in the following examples: "demonstrates a knowledge of methodology," "questions knowledge," "adds knowledge," "creates knowledge," and "simplifies knowledge."

The 12 occurrences of "new" suggest that significant research not only employs new methods but also creates new ways of thinking about reality. In other words, in both its methods and its results, significant research is "new," or different, or perhaps even visionary. Nine of the 12 entries begin with a verb, such as "creates new ways of getting at what's out there," 
Table 1

Characteristics of Significant $\grave{R}$ esearch Word Frequencies: 70 Most Frequent Words

\begin{tabular}{|c|c|c|c|c|c|}
\hline Word & $\begin{array}{c}\text { Word } \\
\text { Frequency }\end{array}$ & $\begin{array}{l}\text { Percent } \\
\text { of Total } \\
\text { Words }\end{array}$ & Word & $\begin{array}{c}\text { Word } \\
\text { Frequency }\end{array}$ & $\begin{array}{l}\text { Percent } \\
\text { of Total } \\
\text { Words } \\
\end{array}$ \\
\hline Knowledge & 13 & 1.23 & Connections & 3 & .28 \\
\hline New & 12 & 1.13 & Theories & $3 \%$ & .28 \\
\hline Based & 11 & 1.04 & Whole & 3 & .28 \\
\hline Data & 9 & .85 & Long & $\mathbf{3}$ & .28 \\
\hline Makes & 7 & .66 & Influence & 3 & .28 \\
\hline Questions & 6 & .57 & Accessible & 3 & .28 \\
\hline Interdisciplinary & 6 & .57 & Problems & 3 & .28 \\
\hline Ideas & 6 & .57 & Issues & 3 & .28 \\
\hline Theory & 5 & .47 & Grounded & 3 & .28 \\
\hline Different & 5 & .47 & Learning & 3 & .28 \\
\hline Beyond & 5 & .47 & Investigation & 3 & .28 \\
\hline Easy & 5 & .47 & Conceptual & 3 & .28 \\
\hline Complex & 5 & .47 & Audience & 3 & .28 \\
\hline Disciplinary & 5 & .47 & Process & 3 & .28 \\
\hline Research & 5 & .47 & Real & 3 & .28 \\
\hline Broad & 4 & .38 & World & 3 & .28 \\
\hline Practical & 4 & .38 & Changes & 3 & .28 \\
\hline Reality & 4 & .38 & Wrong & 3 & .28 \\
\hline Applicable & 4 & .38 & Unique & 3 & .28 \\
\hline Quality & 4 & .38 & Idea & 3 & .28 \\
\hline Life & 4 & .38 & Methods & 3 & .28 \\
\hline Open & 4 & .38 & Related & 3 & .28 \\
\hline Discourse & 4 & .38 & Effects & 3 & .28 \\
\hline Specific & 4 & .38 & Thinking & 3 & .28 \\
\hline Simple & 4 & .38 & Metaphors & 3 & .28 \\
\hline Creates & 4 & .38 & Things & 3 & .28 \\
\hline People & 4 & .38 & Boundaries & 3 & .28 \\
\hline Something & 4 & .38 & Methodology & 3 & .28 \\
\hline Ways & 4 & .38 & Understanding & 3 & .28 \\
\hline Analysis & 4 & .38 & Time & 3 & .28 \\
\hline Messy & 4 & .38 & Important & 3 & .28 \\
\hline Status & 4 & .38 & Inclusive & 3 & .28 \\
\hline Quo & 4 & .38 & Challenges & 3 & .28 \\
\hline Cultural & 4 & .38 & Says & 3 & .28 \\
\hline Intuitive & 4 & .38 & Readers & 3 & .28 \\
\hline
\end{tabular}

"makes a new reality" and "provides new metaphors for thinking about things."

Except for an unusual case ("questions institutionally-based boundaries"), all but one of the 11 uses of the frequently occurring word "based" define significant research as either "based on something" or "something based" as follows: "based on years of practice in the 'real' world"; "based on today's event"; "based on observed data"; "based on strong personal principles - ethical, spiritual"; "hypothesis-based"; "case-study based"; "theory-based"; "minority-group based"; "not empirically based"; and, "empirically based." Although 5 of the 11 occurrences of "based" appear in the methodology category as might be expected, the other 6 occurrences are widely distributed, suggesting that in a number of respects significant research requires a solid basis. It is also interesting to observe that 5 occurrences have to do with basing research in the "real" world (for example, "based on observed data"; "case-study based").

All 7 occurrences of "makes" imply that significant research is dynamic rather than static. In every case, "makes" is an initial verb, as seen here: "makes a difference"; "makes the difficult accessible"; "makes something invisible visible"; "makes sense of stats"; "makes a gestalt"; "makes surprising connection"; "makes a new reality." A similar arrangement occurs in 4 of the 6 occurrences of "questions": "questions institutionally based boundaries of knowledge"; "questions capitalism (for example, established beliefs)"; "adds questions, not just answers"; and, "generates questions not easily resolved."

Three of the 6 appearances of "ideas" describe significant research as an end product, for example, significant research is compressed so that "every sentence is filled with ideas" and significant research uses "commonly understood cultural ideas to present unknown ideas." The other entries imply that research output has an impact on society (for example, it has a "place in the history of ideas" and it presents "ideas that stir you up"). In other words, significant research is an active agent. Also suggesting action, some instances of the word "interdisciplinary," which appears 6 times, occur with verbs, for example, significant research "has broad application (illuminates a whole range of questions/interdisciplinary)." Interestingly enough, of the 293 individual entries in the text as a whole, over half include verbs (54\%), suggesting that a central characteristic of significant research is that it is active and dynamic.

\section{Relationships Among the Frequently Appearing Words}

Further "zooming-in" was achieved using semantic network analysis to plot the most frequently occurring words in a multidimensional space. We began with the 40 most frequently occurring words, a number that is an appropriate starting point since outcomes from this 
kind of analysis become visually indecipherable if too many factors are analyzed. The number of words was further reduced by reviewing a series of plots and systematically removing less interpretatable words until we could produce a plot that was "readable," that is, a plot in which multiple words did not hold the same location. This "right-sizing" effort reduced the number of words to 29, with each word occupying its own space.

Our resulting network analysis is a two-dimensional plot or "perceptual map" of the most frequently occurring 29 words associated with significant research, as shown in Figure 1.

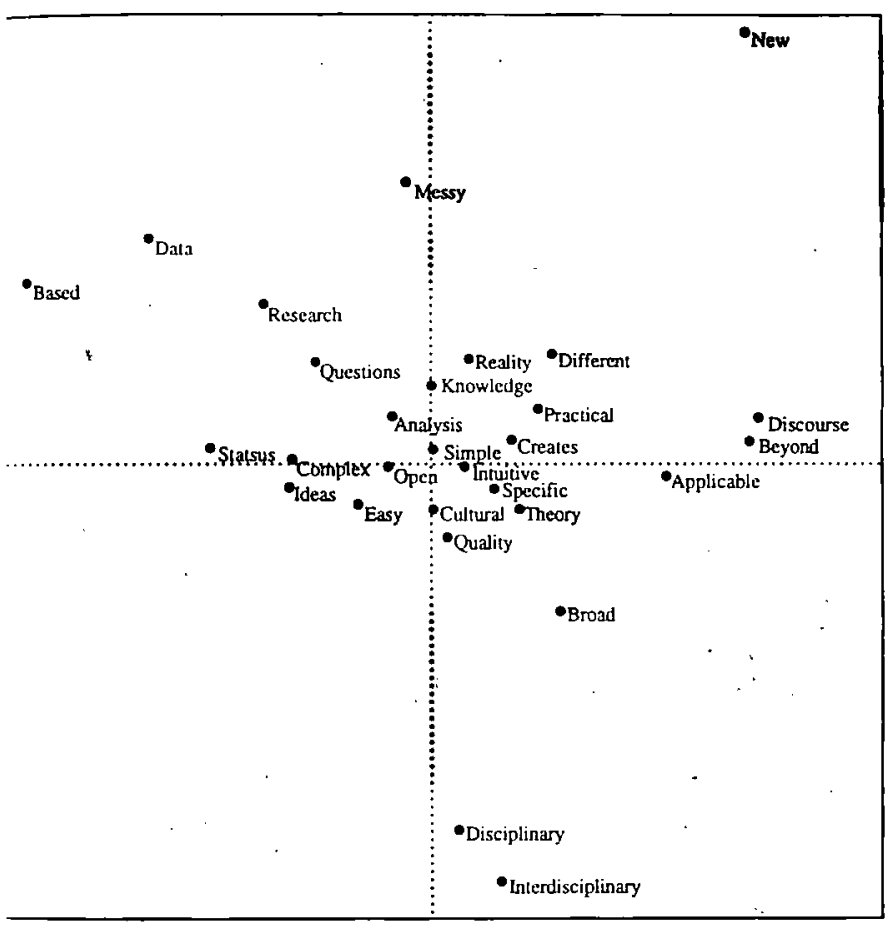

Figure 1. Two-Dimensional Network Analysis of Characteristics of Significant Research

Having generated such a plot, the chore of the researcher is then to interpret it. One way to interpret such plots is to examine each quadrant for words that are close together spatially and that can be logically associated in some way. In this instance, three of the quadrants can be typified using this process: the top left quadrant emphasizes a data-based, research-question aspect of significant research: the top right quadrant includes one verb, "creates." to which the other words can be readily associated to produce the following: creates knowledge, creates reality, and creates something different and new that is simple and practical yet beyond discourse; the bottom right quadrant identifies various dichotomies, namely that research is applicable and theoretical, specific and broad, intuitive (or individual) yet possessing a cultural (or social) quality.

Another useful way to interpret the two-dimensional plot is to collect the words into meaningful descriptive phrases. Starting with the top left quadrant and moving clockwise, words can be associated to form the following statement:

Significant research is data-based, messy, new, beyond discourse, and both disciplinary and interdisciplinary.

Moving toward the cluttered center, interpretation is less clean, although associations can be drawn. One reading of the plot suggests that significant research:

- involves research questions and analysis

- provides knowledge about reality that is different... and new ("new" is obviously an outlier)

- creates simple and practical knowledge about reality

- involves specific theory and intuition

- has a cultural quality

- is open and makes complex ideas easy

- questions the status quo

Next we wondered if the characteristics of significant research that emerged from the two-dimensional network analysis would co-occur in an analysis of another kind. Would some of the same characteristics emerge? To address this question, we compared results from the dimensional plot with results from a hierarchical cluster analysis performed on the 70 most frequently occurring words. This cluster analysis produced a dendogram (Rice \& Danowski, 1993) shown in Table 2 that provided another way of looking at how the words may be associated.

In a dendogram. word clusters are indicated by their side-by-side juxtaposition. The height of the arrows at the bottom of each word entry suggests the strength of the word relationships (much like the volume indicator on a CD sound system) and indicates what words cluster together. Words that are not strongly associated can be dropped. In forming clusters based on a dendogram, the order in which the clustered words are eventually arranged is determined by the researcher.

We interpreted the dendogram (moving from left to right) to form the following word clusters to describe significant research:

makes ideas easy (ideas-easy)

specific analysis

interdisciplinary and disciplinary

influenced by theory (theory-influence)

beyond discourse

grounded in practical problems (practical-problems-

grounded)

real world

something unique

new knowledge

messy methodology

data-based

questions what is wrong with the status quo (statusquo-questions-wrong) 
Table 2

Hierarchical Cluster Analysis Dendogram: 70 Most Frequent Words

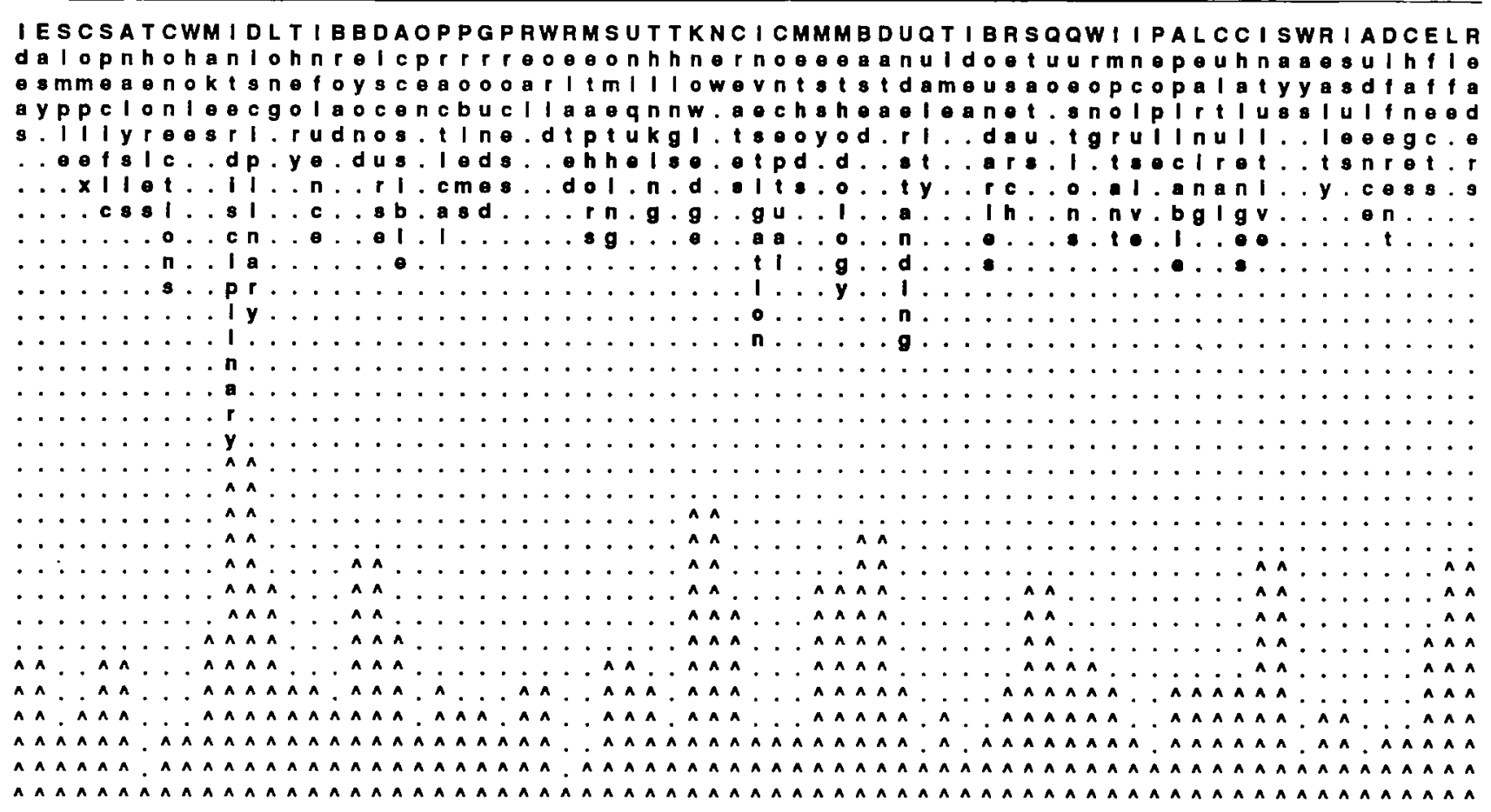

applicable to learning and challenges culture

(applicable-learning-cultural-challenges)

says something intuitive (intuitive-says)

addresses issues in reality (reality-issues)

Finally, by comparing the interpretative lists that resulted from the cluster and the network analyses we were able to match and merge characteristics that were similar (for example, "involves research questions and analysis" and "specific-analysis" were joined to form "involves research questions and specific analysis"). In formulating these merged characteristics, we added initial verbs in order to capture the notion that significant research is an active process, a notion strongly suggested by our contextual analysis. We then categorized these characteristics under three headings that typify the content in the three quadrants from the dimensional network analysis. The resulting list of merged characteristics is below:

\section{Data-Based}

Involves research questions and specific analysis

Is grounded in practical problems of the "real" world

Beyond Discourse

Is open; questions the status quo

Makes complex ideas easy to understand

Creates simple and practical knowledge about reality

Provides new knowledge about reality - something unique

\section{Dichotomy}

Employs method, but is nonetheless messy (as in, there is method to the madness?)

Is influenced by theory and intuition

Is both disciplinary and interdisciplinary

Has a cultural quality, yet challenges culture

Since this list of characteristics resulted from comparing the results of several kinds of analysis of the most frequently occurring words in the original list created by Think Tank participants, it can be called a summary of ideas that researchers repeated in the course of their discussions. It represents a list of ideas that dominated the discussion; ideas that had thematic force.

Systematically reducing a list of 293 characteristics of significant research to a tripartite of 10 items was by no means as awesome a task as trying to capture the essence of a corporate culture in a mission statement, although neither is easy and the end product can seem a little thin. Statements resulting from such efforts cán be meaningful, however, if they identify themes that represent a shared understanding. One reality check of a corporate mission statement is to listen for parallel themes in the stories employees tell or similar words they use to describe their company. To check the tripartite of characteristics of significant research we looked for parallels in recent informal published 
discussions among Association members. Here, in the interest of space, we provide one example.

Dulek's “Three A's of Acceptance" (1994, p. 61) mirrors the tripartite of characteristics of significant research remarkably. Dulek explains that research must be authentic or employ a legitimate methodology to answer relevant research questions, much like the "Data-Based" category above. Then there is the need to be affluent or expansive, rich with new knowledge, and provide a wealth of new insights about ideas previously known, recalling "Beyond Discourse." And, finally Dulek talks about research as meeting an air of excitement; in other words, it must evoke a sense of passion and sense that the researcher is seeking additional information about the topic, a notion that piggybacks nicely with the "Dichotomy" category (see also Smelzter's nine criteria for "good communicationrelated research" (1993, p. 187) compiled from discussions with three journal editors). The parallel with Dulek's 3 A's and the fact that the tripartite itself consists of most-often-associated words begins to suggest that we may have a shared vision as to the characteristics of significant research.

\section{REFERENCES}

Carley, K. M., \& Kaufer, D. S. (1993). Semantic connectivity: An approach for analyzing symbols in semantic networks. Communication Theory, 3, 183-213.

Davis, M. S. (1971). That's interesting! Social Science, 1, 309 344.

Dulek, R. (1994). The three D's of denial; the three A's of acceptance. Bulletin of the Association for Business Communication, 57(2), 61-62.

Krippendorff, K. (1980). Content analysis: An introduction to its methodology. Beverly Hills, CA: Sage.

Rice, R. E., \& Danowski, J. A. (1993). Is it really just like a fancy answering machine? Comparing semantic networks of different types of voice mail users. The Journal of Business Communication, 30, 369.397.

Smeltzer, L. R. (1993). Emerging questions and research paradigms in business communication research. Journal of Business Communication, 30, 181-198.

Woelfel, J. (1990). CATPAC (Version 3.0) [Computer software]. Royal Oak, MI: Terra Research \& Computing.

\section{Your ABC Directory for 1995}

should have reached you by mid-April.

Chock-full of valuable information. Immediately useful.

Did you check your own entry? (Of course you did.) Is it correct?

If yours isn't, how can you trust the addresses for others?

What to do if something is wrong? Get in touch with the ABC home office. Talk to Maggie Abam or send her a card. She's the keeper of information par excellence. She'll make things right.

Because she wants the next issue to be completely right. So you have to provide full and correct and current data. We'll all thank you. 